Reprod. Nutr. Dévelop., 1985, 25 (6), 1047-1060.

\title{
Métabolisme protéique de l'agneau nouveau-né. - I. Conséquences de l'ingestion de colostrum sur la glycémie, la gammaglobulinémie et les teneurs en acides aminés libres des tissus.
}

\author{
P. PATUREAU-MIRAND, O. BERNARD, J. PRUGNAUD, D. LEVIEUX $\left({ }^{*}\right)$, \\ R. PION
}

avec la collaboration technique de G. BAYLE, F. BARRE, M. F. DEVINS (*), M. SALLAS, A. SELLE et M. C. VALLUY

Laboratoire d'Etude du Métabolisme azoté

(*) Laboratoire des Maladies nutritionnelles.

I.N.R.A., Theix, 63122 Ceyrat, France.

Summary. Protein metabolism in the newborn lamb. I. Effect of colostrum feeding on glucose, urea, $\lg G_{1}$ and free amino acids in blood and tissues.

Ten newborn lambs were divided into two groups at birth. Five of them were fed hourly with cow colostrum; the others (unfed lambs) were given saline. Jugular blood levels of glucose, urea, free amino acids and $\mathrm{IgG}_{1}$ were measured during the 10-hour period following birth. The lambs were then exsanguinated under pentobarbital anaesthesia. Free amino acid levels were determined in liver, muscle (longissimus dorsi) and skin. The glycogen contents of the liver and whole body were measured. The $\operatorname{lgG}_{1}$ levels were determined in the intestinal wall and in the contents of the abomasum and small intestine.

The blood glucose levels increased after birth in both groups and did not differ significantly in either group. After colostrum feeding, hepatic glycogen and blood urea concentrations were higher in fed than in unfed lambs. Blood free essential amino acid levels which increased after birth in the fed group, were higher in that group than in the unfed one. Nonessential free amino acid levels remained nearly constant throughout the experimental period and did not differ significantly in either group (fig. 2). Free threonine and valine in the liver, skin and muscle were higher in the colostrum-fed lambs than in the others. IgG levels increased after birth in the blood of the fed lambs and seemed to be closely related to the intestinal contents of these compounds (fig. 3). The amount of blood free essential amino acids provided by the hydrolysis of colostral proteins was estimated at about $43 \%$ of the amount entering the small intestine.

\section{Introduction.}

Le colostrum fournit les nutriments nécessaires à la survie et au développement du nouveau-né au cours de ses premières heures ; il se caractérise, en particulier, par sa richesse en protéines (immunoglobulines principalement) dont la digestion, plus ou moins partielle chez les ruminants, alimente l'organisme en pro- 
téines intactes ou peu dégradées (Bush et Staley, 1980) et en acides aminés. Le lactose du colostrum est aussi une source de glucose pour le nouveau-né. Les augmentations de la glycémie (Demigné et al., 1984) et de la gammaglobulinémie (Cabello et Levieux, 1981) après la naissance ont pu être directement reliées à I'ingestion de colostrum ; elles constituent des facteurs importants de survie (Alexander, 1961 ; Demigné et al., 1984) et de résistance des jeunes ruminants aux agressions microbiennes (Roy, 1980). En revanche, il existe peu de données sur le rôle du colostrum dans l'apport d'acides aminés à l'organisme du nouveauné. L'évolution de l'aminoacidémie qui a été décrite au cours des heures qui suivent la naissance chez le rat (Girard et al., 1975), le porcelet (Cuperlovic, 1967 ; Bengtsson, 1972 ; Pégorier et al., 1981) et l'agneau (Grizard, Patureau-Mirand et Tissier, 1979) n'a que très rarement été reliée de façon précise aux quantités de protéines colostrales ingérées (non mesurées dans la plupart des cas). Pour préciser cet aspect du rôle nutritionnel du colostrum, l'étude de l'évolution de l'aminoacidémie a été entreprise chez des agneaux alimentés ou à jeun au cours des 10 premières heures post-natales. En effet, chez le foetus (Slater et Mellor, 1979) ou chez l'agneau préruminant âgé d'une semaine et plus (Bergen et Potter, 1975 ; Patureau-Mirand et al., 1975, 1977 ; Dove, 1978), ce critère peut, dans un certain nombre de cas, être mis en relation étroite avec les fluctuations de l'apport d'acides aminés exogènes provenant, soit du sang maternel, soit de la digestion des protéines alimentaires. L'étude de l'évolution de la glycémie et de la gammaglobulinémie, associée à celle de la composition des matières azotées des digesta, est réalisée pour compléter la description de la digestion du colostrum. Les variations de l'urémie et de l'aminoacidémie ainsi que les teneurs en acides aminés libres de différents tissus sont comparées chez les agneaux alimentés et à jeun pour apprécier les conséquences du jeûne sur l'utilisation métabolique des acides aminés à cet âge. La mesure, chez les mêmes agneaux, de l'activité de la protéosynthèse dans le muscle, le foie et la peau ainsi que l'estimation du flux métabolique de la leucine et de ses principales composantes (synthèse et dégradation des protéines, catabolisme de la leucine) sont présentées dans l'article suivant.

\section{Matériel et méthodes.}

Animaux. - Les 10 agneaux $(8$ de race Limousine et 2 croisés Romanov $x$ Limousin) pesant à la naissance entre 3,6 et $5,0 \mathrm{~kg}$ sont fournis par le Laboratoire de la Production Ovine (I.N.R.A.). Dès leur naissance, à la bergerie, ils sont séparés de leur mère et installés dans une cage placée dans un local ventilé ayant une température constante de $21{ }^{\circ} \mathrm{C}$ et une hygrométrie voisine de $70 \%$; ils sont rapidement séchés et pesés. Un cathéter est alors introduit, sous anesthésie locale, dans chaque veine jugulaire externe.

Alimentation. - Dès 1 heure après leur naissance, ces 10 agneaux sont alimentés toutes les heures au biberon. Cinq d'entre eux reçoivent $50 \mathrm{ml}$ de colostrum de vache à chacun des 9 repas (agneaux alimentés) ; les 5 autres reçoivent $50 \mathrm{ml}$ d'une solution de $\mathrm{NaCl}(9 \%$ ) (agneaux à jeun). Le colostrum, dont les caractéristiques sont rappelées dans le tableau 1, provient de la première traite après vêlage 
d'une seule vache et a été conservé à $-15^{\circ} \mathrm{C}$. Les aliments sont réchauffés à $35-40{ }^{\circ} \mathrm{C}$ avant d'être distribués au biberon.

TABLEAU 1

Composition du colostrum utilisé.

\begin{tabular}{|c|c|c|c|}
\hline $\begin{array}{l}\text { Matière sèche }(\%)(1) \\
\text { Caséine }(\%)\end{array}$ & $\begin{array}{r}25,1 \\
4,7\end{array}$ & $\begin{array}{l}\text { Matières azotées }(\mathrm{N} \times 6,25 \%) \\
\text { Immunoglobulines } \mathrm{G} 1(\%)\end{array}$ & $\begin{array}{r}16,3 \\
7,4\end{array}$ \\
\hline \multicolumn{4}{|c|}{ Acides aminés $(\mathrm{g} \mathrm{p} 16 \mathrm{~g} \mathrm{~N})$ : } \\
\hline A. aspartique & 8,0 & Méthionine & 2,05 \\
\hline Thréonine & 7,35 & Isoleucine & 3,95 \\
\hline Sérine & 8,15 & Leucine & 8,85 \\
\hline A. glutamique & 15,25 & Tyrosine & 5,20 \\
\hline Proline & 7,25 & Phénylalanine & 4,05 \\
\hline Glycine & 3,55 & Lysine & 7,1 \\
\hline Alanine & 3,80 & Histidine & 245 \\
\hline Valine & 6,95 & Arginine & 4,1 \\
\hline Cystine & 2,05 & & \\
\hline
\end{tabular}

(1) \% du produit frais.

Les poids moyens à la naissance (moyenne \pm écart-type) des 5 agneaux (4 limousins et 1 croisé) de chaque lot (alimenté ou à jeun) sont respectivement de $4,00 \pm 0,54$ et $4,14 \pm 0,37 \mathrm{~kg}$; les quantités moyennes ingérées à chaque repas de $51,6 \pm 1,3$ et $49,9 \pm 0,5 \mathrm{~g}$; les variations moyennes du poids vif des agneaux pendant l'expérience de $76 \pm 30$ et $8 \pm 90 \mathrm{~g}$.

Prélèvements. - Un échantillon de sang est prélevé par un des cathéters, $1 \mathrm{~h}$ après la naissance (juste avant le premier repas), puis 4,7 et $10 \mathrm{~h}$ après la naissance, environ $10 \mathrm{~min}$ avant la tétée. Une quantité aliquote de sang prélevé est recueillie dans 7 fois son volume d'éthanol $95^{\circ}$ froid additionné de $2 \%$ de thiodiglycol pour le dosage des acides aminés libres du sang ; ces échantillons sont conservés à $-15^{\circ} \mathrm{C}$ avant analyse. Pour la mesure de la glycémie et de l'urémie, une quantité aliquote du sang prélevé est recueillie dans 2 fois son volume d'acide trichloroacétique (ATC) à $5 \%$. Ces échantillons sont conservés à $+4{ }^{\circ} \mathrm{C}$. Après avoir été pesés de nouveau, les agneaux sont sacrifiés et saignés après anesthésie générale au pentobarbital sodique, $10 \mathrm{~h}$ après leur naissance. Pour étudier leurs teneurs en acides aminés libres, le foie, un muscle (longissimus dorsi) et un fragment de peau préalablement tondue $(20 \mathrm{~g}$ environ), sont alors rapidement prélevés. Le foie est immédiatement refroidi sur la glace, découpé en petits morceaux, lavé avec une solution de $\mathrm{NaCl} 9 \%$ à $4{ }^{\circ} \mathrm{C}$ puis séché. Il est congelé à l'azote liquide moins de $7 \mathrm{~min}$ après le début de la saignée. La peau et le muscle sont eux aussi congelés en moins de 5 et 10 min respectivement. Les contenus de caillette et d'intestin grêle sont recueillis ainsi qu'une fraction moyenne de la paroi de l'intestin grêle ( $20 \mathrm{~g}$ environ). Ces échantillons, congelés à l'azote liquide, sont conservés à $-15^{\circ} \mathrm{C}$. 
Le reste du corps (moins 2 muscles : tensor fasciae latae et supraspinatus) est congelé et broyé à l'azote liquide pour préparer un échantillon homogène.

Analyses. - Les teneurs en acides aminés libres du sang sont déterminées après extraction alcoolique et purification sur résine échangeuse de cations selon la méthode décrite par Pawlak et Pion (1968). Les teneurs en acides aminés libres des tissus sont mesurées après précipitation des protéines à l'ATC $10 \%$ et purification des surnageants sur résine échangeuse d'anions. Les acides aminés libres ainsi extraits du sang ou des tissus sont dosés par chromatographie sur résine échangeuse de cations à l'aide d'un analyseur automatique. La cystine et le tryptophane libres qui ne sont pas extraits quantitativement, ne sont pas dosés ; la méthyllysine n'est pas séparée de la lysine.

La glycémie et l'urémie sont respectivement mesurées selon une méthode à la glucose-oxydase et à la diacétylmonoxime à l'aide d'un analyseur automatique (Michel, 1971). La teneur en glycogène du foie a été déterminée par la méthode décrite par Gire (1976). Les immunoglobulines G1 ont été dosées par immunodiffusion radiale (Mancini, Carbonara et Heremans, 1965) à l'aide d'un antisérum spécifique préparé au laboratoire selon une méthodologie déjà décrite (Levieux, 1974).

Calculs. - Les différences entre lots sont analysées à l'aide d'un test non paramétrique de Fisher de comparaison des valeurs centrales qui ne nécessite aucune hypothèse concernant la distribution des résultats (Lebart, Morineau et Fénelon, 1979). Les relations entre plusieurs variables sont établies par régressions linéaires simples ou multiples (Snedecor et Cochran, 1971). Les compositions en acides aminés des protéines colostrales ou des digesta sont comparées par analyse factorielle des correspondances (Guilloteau et al., 1983). La représentation graphique de cette analyse permet, dans une certaine mesure de visualiser le degré de similitude des compositions en acides aminés des matières azotées comparées puisqu'elles sont repérées sur le plan principal par des points d'autant plus proches que leur composition est semblable.

L'estimation de la quantité d'acides aminés fournie à l'organisme sous forme libre à la suite de l'ingestion de colostrum, que nous appellerons flux sanguin d'acides aminés alimentaires, est réalisée grâce aux relations établies entre le gain de poids, les teneurs en acides aminés indispensables ou semi-indispensables libres du sang et les quantités ingérées en ces composés par des agneaux préruminants de 8 et 21 jours (Patureau-Mirand et al., 1977). Il est possible de considérer les quantités d'acides aminés ingérées comme des approximations de la quantité fournie à l'organisme sous forme libre par l'aliment puisque la digestibilité apparente des protéines ingérées lors de l'expérience qui a servi à établir ces relations est très élevée $194,6 \%$ d'après Thériez, Patureau-Mirand et Molénat, 1977). Ces relations décrivent de façon relativement satisfaisante les variations des teneurs sanguines en thréonine, valine, méthionine, isoleucine, leucine, tyrosine, phénylalanine et lysine puisque le carré du coefficient de corrélation est compris entre 0,85 et 0,96. Dans le cas de l'histidine et de l'arginine, il n'est que de 0,62 . La validité des estimations réalisées avec ces relations a par ailleurs été testée à l'aide des résultats obtenus dans une autre 
expérience (Patureau-Mirand, Thériez et Prugnaud, 1975), en comparant les aminoacidémies mesurées $(x)$ à celles estimées $(y)$. La régression entre les valeurs estimées et mesurées est $y=1,033( \pm 0,044) x+0,036( \pm 0,027), r=0,96$; cela indique que dans leur ensemble, les estimations sont proches des valeurs mesurées.

\section{Résultats.}

Evolution de la glycémie, des teneurs hépatiques en glycogène et de l'urémie (fig. 1). - La glycémie des agneaux des 2 lots, faible et identique $1 \mathrm{~h}$ après la naissance, est significativement plus élevée dès l'âge de $4 \mathrm{~h}$. Bien que plus forte chez les agneaux alimentés, elle n'est cependant pas significativement différente de celle des agneaux à jeun. La teneur en glycogène du foie est significativement plus élevée chez les agneaux alimentés (52 $\mathrm{mg}$ par $\mathrm{g}$ de foie) que chez les agneaux à jeun $(34 \mathrm{mg})$. En revanche, la teneur moyenne en glycogène du reste du corps n'est pas significativement différente chez les agneaux alimentés $(7,0 \mathrm{mg} / \mathrm{g}$ de tissu) et à jeun $(6,65 \mathrm{mg})$. La liaison entre les teneurs en glycogène hépatique et la glycémie est faible et non significative dans le cas de ces 10 agneaux car des glycémies relativement faibles ont été associées dans certains cas à des teneurs en glycogène hépatique élevées.

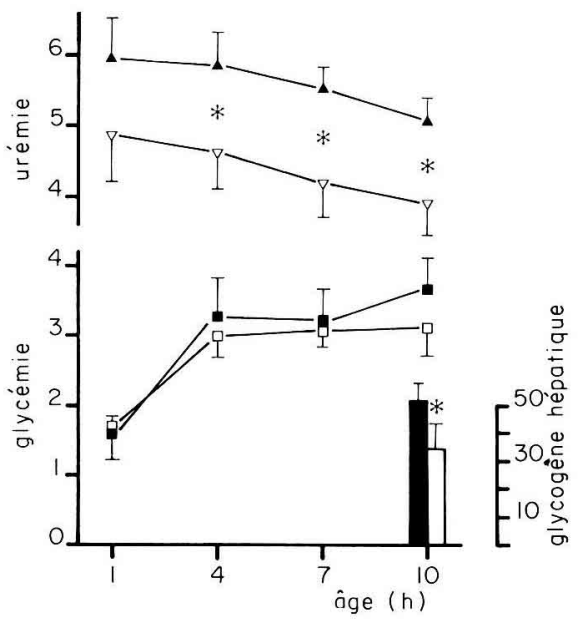

FIG. 1. - Evolution des teneurs sanguines en urée et glucose (mmoles) et concentration en glycogène du foie $(\mathrm{mg} / 100 \mathrm{~g})$ chez les agneaux alimentés $(\boldsymbol{\Delta}, \mathbf{\square})$ ou à jeun $(\triangle, \square)$.

${ }^{*} \mathrm{P}<0,05$.

L'urémie (fig. 1) a été plus élevée dès $1 \mathrm{~h}$ après la naissance dans le sang des agneaux alimentés, bien que de façon non significative. Elle a légèrement diminué entre 1 et $10 \mathrm{~h}$ après la naissance de façon sensiblement parallèle dans les 2 lots ; cependant, elle devient significativement plus faible chez les agneaux à jeun dès $4 \mathrm{~h}$ et le reste jusqu'à $10 \mathrm{~h}$ après la naissance. 
Evolution des teneurs en acides aminés libres du sang (fig. 2). - Chez les agneaux alimentés, les teneurs en acides aminés indispensables tels que valine, isoleucine, leucine, tyrosine et lysine augmentent, surtout entre 1 et $4 \mathrm{~h}$ puis ont tendance à rester stables par la suite. Les teneurs des autres acides aminés indispensables ne varient pas. Chez les agneaux à jeun, les teneurs en tous les acides aminés indispensables diminuent entre 1 et $4 \mathrm{~h}$ puis ne varient plus à l'exception de celles en histidine qui tendent à augmenter pendant les $10 \mathrm{~h}$. II en résulte que les teneurs en thréonine, valine, méthionine $(P=0,07)$, isoleucine, leucine, phénylalanine, tyrosine, lysine, histidine $(P=0,06)$ et arginine sont significativement $(P<0,05)$ plus faibles dans le sang des agneaux à jeun à l'âge de $10 \mathrm{~h}$ que dans celui des agneaux alimentés. Les teneurs en acides aminés non indispensables libres du sang évoluent peu quels que soient les lots. Seules les teneurs en glycine et dans une moindre mesure en glutamine diminuent notablement dans le sang des agneaux des 2 lots, en particulier chez les agneaux alimentés.
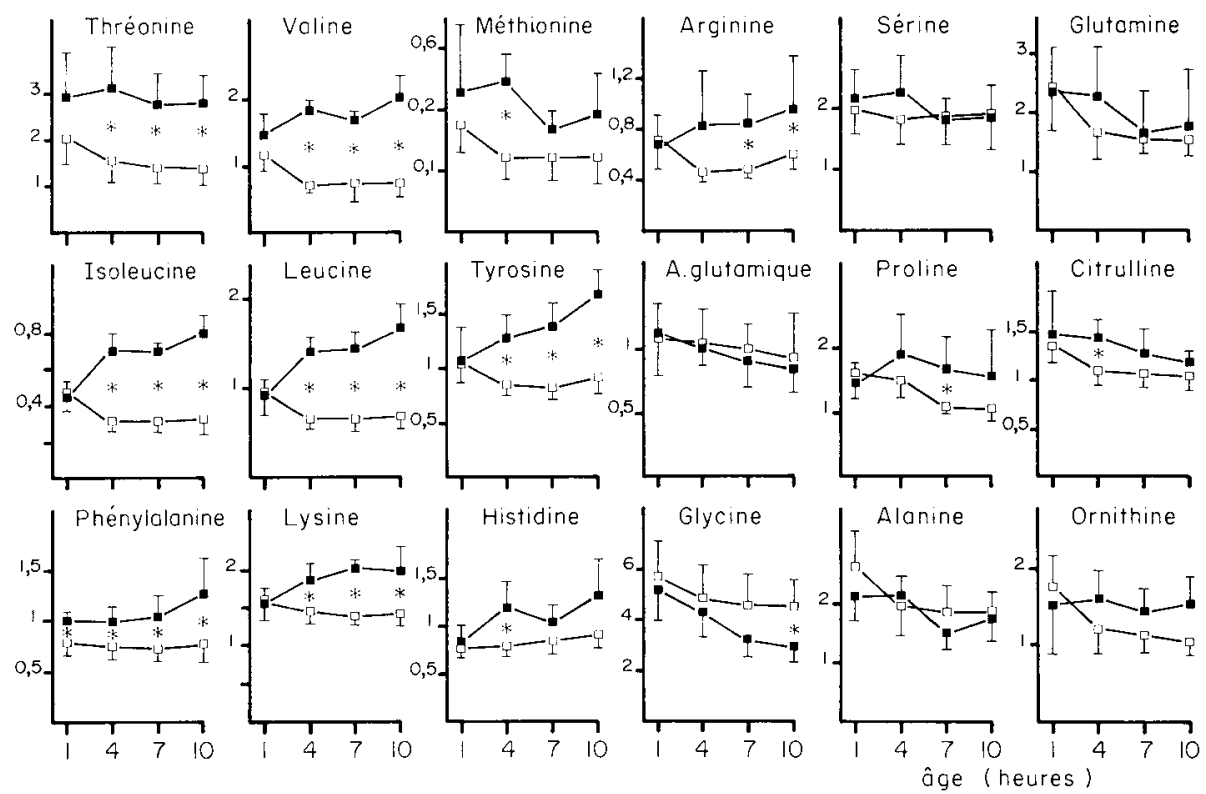

FIG. 2. - Evolution des teneurs en acides aminés libres du sang ( $\mathrm{mg} \% \mathrm{~g}$ ) chez l'agneau nouveau-né alimenté ( $\square$ ) ou à jeun ( $\square$ ).

* Différence significative $(P<0,05)$ entre les teneurs sanguines des agneaux alimentés et celles des agneaux à jeun.

Teneurs en acides aminés libres du foie, du muscle et de la peau (tabl. 2). Dix heures après la naissance, les teneurs en la plupart des acides aminés indispensables sont supérieures dans le foie, le muscle et la peau à celles du sang. Toutefois, les teneurs en acides aminés libres à chaîne ramifiée dans le muscle des agneaux alimentés sont voisines ou inférieures à celles mesurées dans le sang de ces agneaux.

La comparaison des teneurs en acides aminés libres du muscle, de la peau et du foie fait apparaître des situations différentes chez les agneaux alimentés et à 
jeun selon les acides aminés. Les teneurs en thréonine et valine libres sont supérieures dans ces tissus chez les agneaux alimentés à celles mesurées chez les agneaux à jeun. Les teneurs en histidine et tyrosine libres sont plus élevées dans le muscle et la peau des agneaux alimentés ; les concentrations en leucine et dans une moindre mesure en isoleucine libres sont plus élevées dans le foie et la peau des agneaux alimentés que dans ceux des agneaux à jeun ; en revanche, leurs teneurs ne sont pas significativement différentes dans le muscle. Les concentrations en phénylalanine libre ne sont pas différentes dans le muscle ou le foie des agneaux des 2 lots.

\section{TABLEAU 2}

Teneurs en acides aminés libres du sang, du foie, du muscle et de la peau (micromoles/100 g) chez l'agneau nouveau-né alimenté ou à jeun.

\begin{tabular}{lrrrrrrrr}
\hline \multicolumn{1}{c}{ Tissus } & \multicolumn{2}{c}{ Sang } & \multicolumn{2}{c}{ Foie } & \multicolumn{2}{c}{ Muscle } & \multicolumn{2}{c}{ Peau } \\
\hline & \multicolumn{2}{c}{ Alimenté } & A jeun & Alimenté & A jeun & Alimenté & A jeun & Alimenté A jeun \\
Acides aminés indispensables et semi-indispensables : & & & & & \\
Thréonine & 23,2 & $11,7^{* *}$ & 211,3 & $101,4^{* *}$ & 224,6 & $158,9^{*}$ & 80,0 & $42,9^{*}$ \\
Valine & 17,2 & $6,4^{* *}$ & 26,0 & $15,7^{* *}$ & 16,8 & $10,4^{*}$ & 26,0 & $10,8^{* *}$ \\
Isoleucine & 6,3 & $2,5^{* *}$ & 14,1 & 11,2 & 4,7 & 5,5 & 7,9 & $4,8^{*}$ \\
Leucine & 13,4 & $5,4^{* *}$ & 25,5 & $15,9^{*}$ & 10,3 & 9,6 & 19,3 & $9,6^{* *}$ \\
Tyrosine & 8,9 & $5,0^{* *}$ & 10,3 & 8,6 & 15,5 & $10,2^{*}$ & 22,0 & $12,7^{* *}$ \\
Phénylalanine & 7,2 & $4,6^{*}$ & 9,3 & 9,3 & 8,0 & 8,0 & 13,1 & $\left.10,41^{*}\right)$ \\
Lysine & 14,4 & $9,8^{*}$ & 20,9 & 18,9 & 20,4 & 19,0 & 19,2 & $13,2^{* *}$ \\
Histidine & 8,4 & $\left.5,91^{*}\right)$ & 35,3 & 26,9 & 37,4 & $25,3^{*}$ & 19,6 & $14,7^{*}$ \\
Arginine & 5,8 & $3,5^{*}$ & - & - & 46,3 & 55,8 & 18,4 & $13,5^{* *}$ \\
\hline
\end{tabular}

$\left.{ }^{*}\right),{ }^{*},{ }^{* *}$ : différence significative entre agneaux alimentés et agneaux à jeun aux niveaux 10,5 et $1 \%$ respectivement, pour un tissu donné.

Digestion des protéines colostrales. - Au cours de l'expérience, les agneaux alimentés ont ingéré $72 \mathrm{~g}$ de protéines en moyenne dont $28 \mathrm{~g}$ ont été retenus dans la caillette. Une fraction des $34,3 \mathrm{~g} \mathrm{~d}^{\prime} \mathrm{lgG}_{1}$ ingérés se retrouve dans les contenus de caillette $(4,81 \mathrm{~g})$, d'intestin grêle $(4,14 \mathrm{~g})$ et dans la paroi intestinale $(4,43 \mathrm{~g})$; une autre est passée dans le plasma dont les teneurs s'accroissent forte-

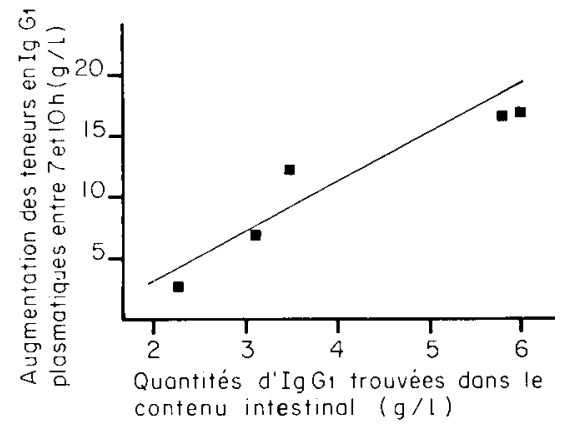

FIG. 3. - Relation entre les variations de la gammaglobulinémie et les quantités $d^{\prime} / g G_{1}$ présentes à $10 \mathrm{~h}$ dans le contenu intestinal. 
ment (tabl. 3). Cette augmentation, variable selon les agneaux, ne paraît pas directement liée à la quantité d'immunoglobulines ingérées non retrouvées dans le tractus digestif. En revanche, elle est étroitement corrélée, notamment entre 7 et $10 \mathrm{~h}$, à la quantité d'immunoglobulines présente dans le contenu intestinal (fig. 3).

TABLEAU 3

Digestion des immunoglobulines colostrales $\left(\lg _{1}\right)$ chez l'agneau nouveau-né.

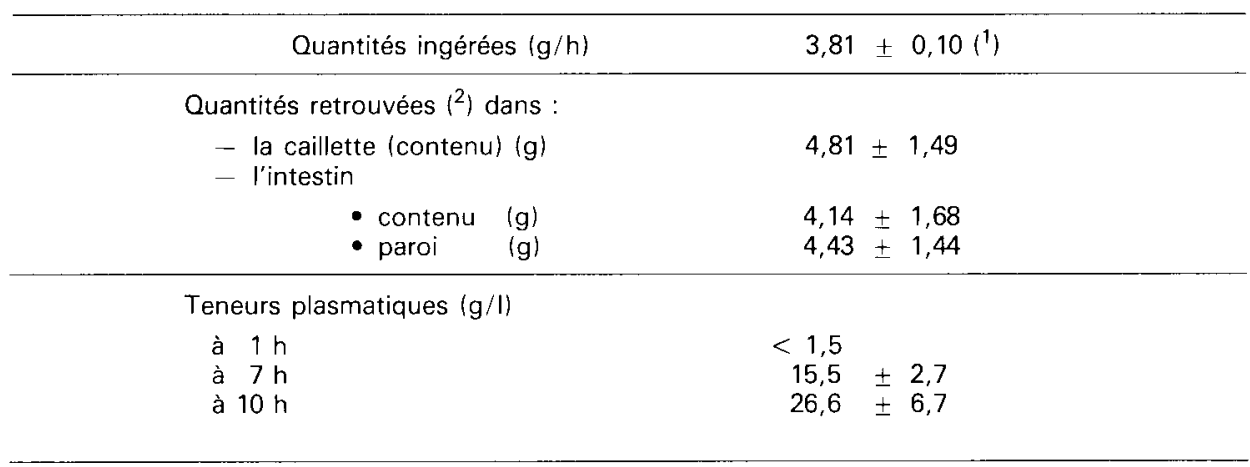

(1) Ecart-type ; $\left({ }^{2}\right)$ à l'âge de $10 \mathrm{~h}$ au moment de l'abattage.

Les compositions en acides aminés des protéines ingérées ou retrouvées dans la caillette ou ayant atteint l'intestin grêle, sont comparées sur la figure 4 à celles des 3 principales protéines colostrales (immunoglobulines, caséine, $\beta$ lactoglobuline). Les contenus de caillette ont une composition en acides aminés plus proche de celle de la caséine que de celle du colostrum ; en revanche, les matières azotées parvenues dans l'intestin ont une composition voisine de celle des immunoglobulines.

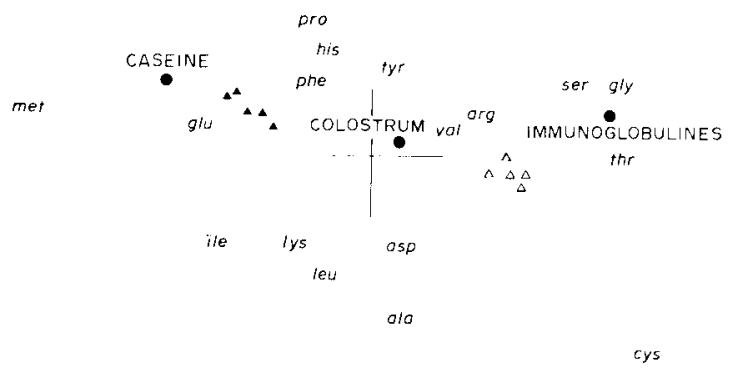

B LACTOGLOBULINE

FIG. 4. - Comparaison des compositions en acides aminés des protéines colostrales (1) $(\bullet)$ et des contenus de caillette $(\mathbf{\Delta})$ et d'intestin grêle $\left({ }^{2}\right)(\Delta)$.

(1) Caséine (Pion et Fauconneau, 1968). Immunoglobulines (G1) (Kickhofen, Hammer et Scheel, 1968) : $\beta$ lactaglobuline (Jenness, 1974). $\left({ }^{2}\right)$ Calculées par différence entre les quantités ingérrées et les quantités retrouvées dans la caillette. 
Enfin, les flux sanguins des acides aminés indispensables alimentai es lestimés selon le calcul indiqué ci-dessus), sont nettement inférieurs aux quantités d'acides aminés du colostrum parvenues dans l'intestin (tabl. 4).

TABLEAU 4

Comparaison des flux d'acides aminés indispensables (') alimentaires dans /'intestin ( ${ }^{2}$ ) et dans le sang $\left(^{3}\right)$ chez l'agneau nouveau-né $(\mathrm{g} / \mathrm{kg} / \mathrm{j})$.

\begin{tabular}{llcc}
\hline \multicolumn{1}{c}{ Acides aminés } & \multicolumn{1}{c}{ Flux intestinal } & Flux sanguin & Rapport (\%) \\
\cline { 2 - 4 } Thréonine & $2,428 \pm 0,278\left(^{4}\right)$ & $0,561 \pm 0,041$ & 23,1 \\
Valine & $1,978 \pm 0,162$ & $0,718 \pm 0,038$ & 36,3 \\
Méthionine & $0,314 \pm 0,043$ & $0,232 \pm 0,039$ & 73,9 \\
Isoleucine & $0,960 \pm 0,069$ & $0,573 \pm 0,031$ & 59,7 \\
Leucine & $2,430 \pm 0,220$ & $1,088 \pm 0,050$ & 44,8 \\
Tyrosine & $1,364 \pm 0,103$ & $0,507 \pm 0,038$ & 37,2 \\
Phénylalanine & $1,010 \pm 0,076$ & $0,595 \pm 0,045$ & 58,9 \\
Lysine & $1,820 \pm 0,185$ & $0,991 \pm 0,097$ & 54,4 \\
Histidine & $0,626 \pm 0,046$ & $0,313 \pm 0,055$ & 50,0 \\
Arginine & $1,160 \pm 0,107$ & $0,507 \pm 0,085$ & 43,7
\end{tabular}

(1) Plus tyrosine ; $\left({ }^{2}\right)$ Flux d'acides aminés alimentaires dans l'intestin = quantité ingérée moins quantité retrouvée dans la caillette; ( $\left.{ }^{3}\right)$ Flux d'acides aminés alimentaires dans le sang = quantités estimées par le calcul présenté en Matériels et Méthodes ; $\left({ }^{4}\right)$ Ecart-type.

\section{Discussion.}

Evolution de la glycémie. - L'augmentation de la glycémie dans les heures qui suivent la naissance a souvent été décrite chez les jeunes ruminants (Edwards, 1970). Chez les agneaux alimentés, elle pourrait résulter de la digestion du lactose du colostrum. Cependant, cet accroissement de la glycémie a aussi été observé chez des ruminants nouveau-nés restés à jeun : veau (Edwards, 1970), agneau (Alexander, 1962). II serait alors la conséquence de la mobilisation des réserves glycogéniques du foie. En effet, les teneurs en glycogène du foie des agneaux alimentés, $10 \mathrm{~h}$ après la naissance, sont voisines de celles mesurées à la naissance par Alexander (1962) : $60 \mathrm{mg} / \mathrm{g}$, à l'aide d'une autre méthode il est vrai. Par contre, celles des agneaux à jeun sont significativement plus faibles. Cependant, la glycogénolyse hépatique ne peut constituer la seule source de glucose de ces animaux dont le taux de renouvellement est important (Warnes, Seamark et Ballard, 1977a). Une gluconéogenèse stimulée dès les premières minutes après la naissance (Warnes, Seamark et Ballard, 1977b) peut aussi contribuer à l'augmentation de la glycémie. II se pourrait toutefois que la participation des acides aminés à cette gluconéogenèse soit modeste à cet âge dans la mesure où elle peut s'apprécier par l'intensité du catabolisme des acides aminés. En effet, la baisse plus rapide de l'urémie après la naissance chez les agneaux à jeun paraît indiquer que le catabolisme des acides aminés $y$ est moins intense que chez les agneaux alimentés. 
Evolution de l'aminoacidémie. - L'augmentation des teneurs en tous les acides aminés indispensables chez les agneaux nouveau-nés alimentés a déjà été observée chez l'agneau (Grizard, Patureau-Mirand et Tissier, 1979), le veau, le chevreau (Patureau-Mirand, 1984) et le porcelet (Pégorier et al., 1981). Etant donné que les concentrations de ces composés (histidine exceptée) tendent à diminuer dans le sang des agneaux restés à jeun pendant la même période, il est probable que l'accroissement observé chez les agneaux alimentés corresponde à la digestion des protéines colostrales.

Digestion des protéines colostrales. - La similitude de composition en acides aminés des contenus de caillette avec la caséine indique que cette dernière, qui coagule sous l'action de la chymosine présente en quantités importantes dans la caillette de l'agneau nouveau-né (Guilloteau et al., 1983), constitue une fraction importante des protéines alimentaires retenues à ce niveau ; cependant elle peut y subir une protéolyse non négligeable (Yvon et al., 1983). Le flux intestinal de protéines serait alors principalement constitué de protéines du lactosérum de colostrum, contenant surtout des immunoglobulines. Le passage dans l'intestin grêle d'immunoglobulines est vérifié par la présence dans le contenu et la paroi de l'intestin de quantités notables $d^{\prime} \lg _{1}$ ayant conservé leur immunoréactivité.

L'augmentation des teneurs plasmatiques en $\operatorname{lgG}_{1}$ des agneaux alimentés est comparable à celle décrite par Cabello et Levieux (1981). La liaison avec les quantités présentes dans le contenu intestinal paraît indiquer que l'un des facteurs de l'absorption de ces composés pendant cette période pourrait être leur vitesse de transit gastrique (Ruckebusch, Dardillat et Guilloteau, 1983).

La digestion et l'absorption de ces protéines pénétrant dans l'intestin se sont accompagnées d'un accroissement des teneurs sanguines en acides aminés indispensables libres. Cependant, étant donné la digestion incomplète des protéines colostrales entrées dans l'intestin grêle, il n'est pas possible d'estimer directement la quantité d'acides aminés d'origine alimentaire qu'elle libère dans l'organisme. Nous avons alors dû employer une méthode d'estimation indirecte de ce flux sanguin d'acides aminés libres d'origine alimentaire qui suppose la vérification de plusieurs hypothèses.

On peut en effet se demander s'il est convenable d'appliquer à l'agneau nouveau-né des relations établies chez des agneaux âgés de 8 et 21 jours. Le fait que l'utilisation de ces relations chez les agneaux nouveau-nés à jeun conduise à des estimations d'acides aminés ingérés non significativement différentes de zéro peut le laisser penser. Une autre difficulté de l'utilisation de ces relations tient à la nécessité de connaître le gain de poids des animaux au moment des prélèvements. Or, il peut paraître illusoire de penser mesurer la variation du poids sur un intervalle de $9 \mathrm{~h}$ pour un agneau donné. Cependant, la moyenne des variations de poids des 5 agneaux alimentés est significative $(200 \mathrm{~g} / \mathrm{j})$ et compatible avec les observations de Villette et Thériez (1983) ; en revanche, cette variation moyenne de poids est quasi-nulle chez les agneaux restés à jeun ce qui est en accord avec les résultats d'Alexander (1962). II semble donc que les estimations des flux sanguins d'acides aminés indispensables d'origine alimentaire déduits de l'utilisation de ces relations puissent être considérées comme des approximations de la contri- 
bution des protéines colostrales à la fourniture d'acides aminés libres à l'organisme.

Le flux sanguin de l'ensemble des acides aminés indispensables ingérés représenterait donc $6,1 \mathrm{~g} / \mathrm{j} / \mathrm{kg}$ ce qui correspond à l'hydrolyse d'environ $0,49 \mathrm{~g} / \mathrm{h} / \mathrm{kg}$ de protéines colostrales. Cette dernière quantité est très inférieure aux $1,89 \mathrm{~g} / \mathrm{h} / \mathrm{kg}$ ingérés au cours des $10 \mathrm{~h}$ de l'expérience mais très supérieure à la quantité d'acides aminés contenus sous forme libre dans le colostrum 11 à $2 \%$ des acides aminés colostraux). Contrairement à ce qui a été suggéré par Cuperlovic (1967) et Bengtsson (1972) pour le porcelet, les variations de l'aminoacidémie chez l'agneau nouveau-né alimenté ne peuvent vraisemblablement pas s'expliquer par la seule absorption des acides aminés libres du colostrum mais la fourniture d'acides aminés par l'hydrolyse des protéines colostrales doit être envisagée. La faible contribution au flux sanguin des acides aminés qui se trouvent en proportion relativement importante dans les IgG (thréonine, valine et tyrosine) peut s'expliquer par la résistance relative de ces protéines à la protéolyse. En revanche, les valeurs élevées du rapport du flux sanguin au flux intestinal pour la méthionine, l'isoleucine et la leucine résultent probablement d'une dégradation plus intense de la caséine et/ou de la $\beta$ lactoglobuline.

Influence de l'ingestion de colostrum sur les teneurs en acides aminés libres des tissus. - La différence des concentrations en acides aminés indispensables libres du sang observée entre les agneaux alimentés et à jeun ne se retrouve pas de façon constante dans les tissus pour tous ces composés. En effet, si dans la peau, les écarts observés sont comparables à ceux constatés dans le sang (phénylalanine exceptée), il $n^{\prime}$ en est pas de même dans le foie et le muscle. Dans le foie, les différences de concentrations ne subsistent que pour la thréonine qui, chez les ovins, est en concentration élevée et pour la valine et la leucine qui sont peu catabolisées dans le foie (Odessey et Goldberg, 1972). En revanche, dans le muscle, il n'y a pas de différence de concentrations en isoleucine et leucine libres chez les agneaux alimentés et à jeun alors que celle en valine est importante. Cela paraît résulter de l'absence d'une diminution des teneurs en leucine et isoleucine dans le muscle des agneaux à jeun, proportionnelle à celle du sang et/ou d'une augmentation limitée des teneurs en ces 2 composés dans le muscle des agneaux alimentés. La réduction de la protéosynthèse dans le muscle des agneaux à jeun (Patureau-Mirand et al., 1985) peut contribuer à expliquer l'absence d'une réduction des teneurs en plusieurs acides aminés libres comparable à celle du sang (valine, isoleucine, leucine, phénylalanine, lysine). Un catabolisme musculaire actif de l'isoleucine et surtout de la leucine, tel qu'il est décrit chez le rat (Odessey et Goldberg, 1972) pourrait limiter l'augmentation des teneurs en ces acides aminés dans le muscle des agneaux alimentés. Cependant, selon Lindsay (1982), la transamination des acides aminés à chaîne ramifiée serait moins active dans le muscle des ruminants; cela n'est toutefois pas confirmé par les travaux de Wijayasinghe, Milligan et Thompson (1983). 


\section{Conclusion.}

L'étude de l'évolution de l'aminoacidémie chez des agneaux alimentés ou à jeun au cours des premières heures qui suivent la naissance met en évidence le rôle du colostrum dans l'apport d'acides aminés indispensables à l'organisme du nouveau-né. Toutefois, les quantités d'acides aminés indispensables ainsi apportées sont sensiblement inférieures aux quantités ingérées du fait de l'absorption de protéines non hydrolysées (immunoglobulines notamment). On peut alors se demander quelles sont les conséquences de cet apport d'acides aminés, mais aussi d'autres nutriments (glucose) sur le métabolisme protéique du nouveau-né. Si l'étude des variations de l'urémie et des teneurs en acides aminés libres des tissus peuvent fournir des indications, celles-ci doivent être précisées par l'estimation des flux d'acides aminés selon les principales voies du métabolisme protéique (protéosynthèse, protéolyse, catabolisme des acides aminés).

Reçu en février 1985.

Accepté en septembre 1985.

Remerciements. - Nous remercions MM. A. Talmant et G. Monin de la Station de Recherches sur la Viande (I.N.R.A.) pour leur contribution au dosage du glycogène.

\section{Références}

ALEXANDER G., 1961. Temperature regulation in the newborn lamb. III. Effect of environmental temperature on metabolic rate, body temperatures, and respiratory quotient. Aust. J. agric. Res., 12, 1152-1174.

ALEXANDER G., 1962. Energy metabolism in the starved newborn lamb. Aust. J. agric. Res., 13, 144-164.

BENGTSSON G., 1972. Sow colostral free amino acids and their importance for the newborn piglet. Acta vet. scand., 63, 672-680.

BERGEN W. G., POTTER E. L., 1975. Effect of dietary protein level on plasma and tissue free amino acid concentrations in nursing lambs. J. anim. Sci., 40, 789-794.

BUSH L. J., STALEY T. E., 1980. Absorption of colostral immunoglobulins in newborn calves. J. Dairy Sci., 63, 672-680.

CABELLO G., LEVIEUX D., 1981. Absorption and half-life of bovine, caprine and ovine IgG in the newborn lamb. Effect of experimental prematurity and endocrine factors. Ann. Rech. vét., 12, 421-429.

CUPERLOVIC M., 1967. Free amino acids in the blood plasma of newborn piglets before and after first intake of colostrum. Acta vet. scand., 8, 217-227

DEMIGNÉ C., CHARTIER F., RÉMÉSY C., RAYSSIGUIER Y., 1984. Etude de l'évolution du métabolisme postnatal chez l'agneau et le veau, 189-202. In JARRIGE R., Physiologie et pathologie périnatales chez les animaux de la ferme, I.N.R.A, Paris.

DOVE H., 1978. Utilisation of amino acids by pre-ruminant lambs. III. The influence of age and the dietary proportions of essential and non-essential amino acids on the levels of urea, ammonia and amino acids in the blood plasma of milk-fed lambs. Aust. J. agric. Res., 29, 145-160.

EDWARDS A. V., 1970. Carbohydrate metabolism in young animals, 180-198. In PHILLIPSON A. T., Physiology of digestion and metabolism in the ruminant. Oriel Press, Newcastle-upon-Tyne.

GIRARD J. R., GUILLET I., MARTY J., MARLISS E. B., 1975. Plasma amino acid levels and development of hepatic gluconeogenesis in the newborn rat. Am. J. Physiol., 229, 466-473. 
GIRE P., 1976. Mise au point d'une technique de dosage du glycogène musculaire et application à l'étude de l'influence du stress du transport sur la glycogénolyse post mortem. Th. Doct. $3^{e}$ cycle, Clermont-Ferrand, $107 \mathrm{p}$.

GRIZARD J., PATUREAU-MIRAND P., TISSIER M., 1979. Influence du niveau des apports énergétiques pendant la fin de la gestation sur l'insulinémie et l'aminoacidémie des brebis et de leurs agneaux. Ann. Biol. anim. Bioch. Biophys., 19, 199-205.

GUILLOTEAU P., CORRING T., GARNOT P., MARTIN P., TOULLEC R., DURAND G., 1983. Effects of age and weaning on enzyme activities of abomasum and pancreas of the lamb. J. Dairy Sci, 66, 2373-2385.

JENNESS R., 1974. The composition of milk, 3-107. In LARSON B. L., SMITH V. R., Lactation, a comprehensive treatise, vol. 3, Nutrition and biochemistry of milk/maintenance. Acad. Press, New York.

KICKHOFEN B., HAMMER D. K., SCHEEL D., 1968. Isolation and characterisation of $\gamma \mathrm{G}$ type immunoglobulins from serum and colostrum. Hoppe-Seyler's Z. Physiol. Chem., 349, 17551773.

LEBART L., MORINEAU A., FENELON J. P., 1979. Traitement des données statistiques, 305-328. Dunod, Paris.

LEVIEUX D., 1974. Immunoglobulines bovines et brucellose. 1. Purification des immunoglobulines et préparation de leurs antisérums spécifiques. Ann. Rech. vét., 5, 329-342.

LINDSAY D. B., 1982. Relationships between amino acid catabolism and protein anabolism in the ruminant. Fed. Proc., 41, 2550-2554.

MANCINI G., CARBONARA A. O., HEREMANS J. F., 1965. Immunochemical quantitation of antigens by single radial immunodiffusion. Immunochemistry, 2, 235-254.

MICHEL M. C., 1971. Analyse quantitative de quelques substances azotées et glucidiques en milieu biologique. Essai de rationalisation. Th. Doct. Univ., Clermont-Ferrand, 97 p.

ODESSEY R., GOLDBERG A. L., 1972. Oxidation of leucine by rat skeletal muscle. Am. $J$. Physiol., 223, 1376-1383.

PATUREAU-MIRAND P., 1984. Métabolisme azoté chez le fœtus et le nouveau-né, 231-246. In JARRIGE R., Physiologie et pathologie périnatales chez les animaux de la ferme. I.N.R.A, Paris.

PATUREAU-MIRAND P., BERNARD O., PRUGNAUD J., ARNAL M., $1985 . \quad$ Métabolisme protéique de l'agneau nouveau-né. 2. Influence de l'alimentation sur les flux et le taux de renouvellement des protéines. Reprod. Nutr. Dévelop., 25, 1061-1073.

PATUREAU-MIRAND P., THÉRIEZ M., PRUGNAUD J., 1975. Influence de la nature des protéines alimentaires sur l'aminoacidémie de l'agneau préruminant. Ann. Biol. anim. Bioch. Biophys., 15. 95-101.

PATUREAU-MIRAND P., THÉRIEZ M., PRUGNAUD J., PION R., 1977. Influence du taux protéique et de la composition en acides aminés de l'aliment d'allaitement sur l'aminoacidémie de l'agneau préruminant. Ann. Zootech., 26, 315-328.

PAWLAK M., PION R., 1968. Influence de la supplémentation des protéines du blé par des doses croissantes de lysine sur la teneur en acides aminés libres du sang et du muscle du rat en croissance. Ann. Biol. anim. Bioch. Biophys., 8, 517-530.

PÉGORIER J. P., DUÉE P. H., ASSAN R., PÉRET J., GIRARD J., 1981. Changes in circulating fuels, pancreatic hormones and liver glycogen concentration in fasting or suckling newborn pigs. J. develop. Physiol., 3, 203-217.

PION R., FAUCONNEAU G., 1968 . Les ressources mondiales actuelles et futures en protéines disponibles pour l'alimentation de I'homme et leurs possibilités d'utilisation, 3-59. In /sotopes studies on the nitrogen chain. Int. at. Energy Agency, Vienne.

ROY J. H. B., 1980. Factors affecting susceptibility of calves to diseases. J. Dairy Sci., 63, 650-664.

RUCKEBUSCH Y., DARDILLAT C., GUILLOTEAU P., 1983. Development of digestive functions in the newborn ruminant. Ann. Rech. vét., 14, 360-374.

SLATER J. S., MELLOR D. J., 1979. Concentrations of free amino acids in maternal and fetal plasma from conscious catheterised ewes during the last five weeks of pregnancy. Res. vet. Sci., 26, 296-301.

SNEDECOR G. W., COCHRAN W. G., 1971. Méthodes statistiques. A.C.T.A., Paris, 649 p.

THERIEZ M., PATUREAU-MIRAND P., MOLENAT G., 1977. Allaitement artificiel de l'agneau. VI. 
Comparaison de différentes teneurs en matières azotées du lait de remplacement. Ann. Zootech., 26, 297-313.

VILLETTE Y., THERIEZ M., 1983. Influence du poids à la naissance sur les performances d'agneaux de boucherie. I. Niveau d'ingestion et croissance. Ann. Zootech., 30, 151-168.

WARNES D. M., SEAMARK R. F., BALLARD F. J., 1977a. Metabolism of glucose, fructose and lactate in vivo in chronically cannulated foetuses and in suckling lambs. Biochem. J., 162, 617-626.

WARNES D. M., SEAMARK R. F., BALLARD F. J., 1977b. The appearance of gluconeogenesis at birth in sheep. Activation of the pathway associated with blood oxygenation. Biochem. J., 162, 627-634.

WIJAYASINGHE M. S., MILLIGAN L. P., THOMPSON J. R., 1983. In vitro degradation of leucine in muscle, adipose tissue, liver, and kidney of fed and starved sheep. Biosci. Rep., 3, 11331140.

YVON M., PELISSIER J. P., GUILLOTEAU P., TOULLEC R., 1983. Digestion des protéines du lait dans la caillette du veau préruminant. IVe Symp. int. Métabolisme et nutrition azotés. Vol. 2 , 357-359. I.N.R.A Publ. Versailles. 